\title{
A checklist of Tanzanian myxomycetes
}

\author{
TARJA UKKOLA
}

UKKOLA, T. 2000: A checklist of Tanzanian myxomycetes. - Karstenia 40: 189194. Helsinki. ISSN 0435-3402

\begin{abstract}
A brief review of the Tanzanian myxomycete research is made. An annotated checklist is given of all the names by which Tanzanian myxomycetes are listed in the literature. The check-list includes all myxomycete taxa known to be reported from Tanzania, East-Africa. The known biota of myxomycetes in Tanzania now includes 124 species and four varieties.
\end{abstract}

Key words: Africa, checklist, myxomycetes, Tanzania.

Tarja Ukkola, Department of Ecology and Systematics, P.0. Box 47, FIN-00014 University of Helsinki, Finland

\section{Introduction}

The first known records of the Tanzanian myxomycetes are those of some German researchers in the beginning of the $20^{\text {th }}$ century. In 1891-1918 the area of present Tanzania was a colony of Germany (Tanganyika). In 1902 the Germans founded a research station called "Das BiologischLandwirtschaftliche Institut Amani", located in the submontane rain forest of the East Usambara mountains, NE of present Tanzania (Iversen 1991). Amani was the biggest research centre in the German East Africa, and was visited by many biologists. The first records of Tanzanian myxomycetes also originate from there. Hennings (1905) mentioned three species of myxomycetes in his list of "Fungi Africae orientalis. III". The first more comprehensive study of Tanzanian myxomycetes is that of Eichelbaum (1906). He reported 22 myxomycete specimens representing 17 species, mainly from Amani. Most of Eichelbaum's collections were possibly deposited in Berlin, and were destroyed in World War II. Only five specimens (representing three species) are still left in Hamburg (HBG), and have been studied by me. In 1907, Hennings cited four myxomycete species in his publication "Fungi Africae orientalis. IV". These collections, however, belong to those already reported by Eichel- baum. In a study of fungi growing on dung, Schmidt (1913) lists two myxomycete species, also from Amani.

In 1916, the Amani station was occupied by British forces, and after the war in 1914-1918 Tanganyika was controlled by Britain as a mandate territory (Iversen 1991). There was a long pause in the myxomycete studies, until some records of myxomycetes from Tanzania were published by Eliasson and Lundqvist (1979). They reported six species (eight specimens) of myxomycetes developed in moist chamber cultures on the dung of herbivores (Eliasson \& Lundqvist 1979). The myxomycetes of Tanzania were studied more intensively in 1988-1995 during several expedition trips made by the Department of Ecology and Systematics, University of Helsinki, Finland. The results were published in the following papers: Härkönen and Saarimäki (1991, 1992, 1994), Ukkola and Härkönen (1996), Ukkola et al. (1996), Ukkola (1998a,b,c).

Although many vegetation zones in different parts of Tanzania were visited during the studies made by the group from the University of Helsinki, our knowledge of the distribution and diversity of myxomycete species in Tanzania is far from complete. The most intensively studied area 
even today is the place from where the first records originate: Amani in the East Usambara Mountains. Tanzania is a large country with variable climate and vegetation, and there are still many unexplored habitats, which are potentially favourable for myxomycetes. The still remaining lowland rain forests, moss forests (cloud/elfin forests) and bamboo thickets in the high mountains, the trees in miombo woodlands, and semideserts are examples of the vegetation types where more intensive studies are needed.

The following check-list includes all taxa reported until now from Tanzania. The check-list includes 125 presently valid species and four varieties; 44 of these have been observed only in moist chamber cultures prepared with bark from living trees, decayed wood, plant debris or dung. The occurrence of Hemitrichia clavata (Pers.) Rostaf., is, however, doubful in Tanzania.

The taxa reported in Ukkola et al. (1996) and Ukkola (1998a,b) with descriptions and illustrations, but identified only to the genus level are not included in the check-list.

\section{Criteria followed}

The taxa are listed in alphabetical order. The check-list includes all the names used for Tanzanian myxomycetes in the literature. Valid names are printed in bold type (in italic type in the notes), synonyms or not accepted combinations are in ordinary type. The numbers in bold after the species names refer to the publications in which they are reported. Species reported in the literature but without representative herbarium material are marked with an asterisk (*). The taxa with doubtful identity are in parentheses.

The nomenclature follows mainly Martin and Alexopuolos (1969), Martin et al. (1983) or, especially concerning some members of the Stemonitales, Nannenga-Bremekamp $(1967,1991)$.

The following works have been used for checking the sites and years of publication of pre-1968 taxa: Rostafinski $(1874,1875,1876)$, Lister $(1911,1925)$, and Martin and Alexopoulos (1969). The corresponding information for later taxa is based on the original publications, and on Martin and Alexopoulos (1969), NannengaBremekamp (1991), Neubert et al. $(1993,1995)$ and Ing (1999).

Authors are abbreviated according to Kirk and Ansel (1992).

\section{The check-list}

Arcyria affinis Rostaf., Mon.:276. 1875, emend. Nann.-Bremek., Proc. Kon. Nederlandse Akad. Wetensch. C 71:39. 1968. 3, 4

Arcyria aff. afroalpina Rammeloo, Bull. Jard. Bot. Nat. België 51:229. 1981. 9

Arcyria cinerea (Bull.) Schum., Enum. Pl. Saell.: n.1480. 1803. $6 \rightarrow$ Arcyria cinerea (Bull.) Pers.

Arcyria cinerea (Bull.) Pers., Syn. Fung.:184. 1801. 3, $4,8,9,12$. Note 1 .

Arcyria denudata (L.) Wettst., Verh. Zool.-Bot. Ges. Wien 35: Abh.: 535. 1886. 3, 4, 9, 12

Arcyria globosa Schwein., Schr. Nat. Ges. Leipzig 1:64. 1822. 3, 4

Arcyria insignis Kalchbr. \& Cooke, in Grevillea 10:143. 1882. 3, 4, 9

Arcyria minuta Buchet, in Patoullard, Mém. Akad. Malgache 6:42. 1927. 9, 12

Arcyria obvelata (Oeder) Onsberg, Mycologia 70:1286. 1978. 3, 4, 9, 12

Arcyria pomiformis (Leers) Rostaf., Mon.:271. 1875. 9, 12

Arcyria punicea Pers., in Roemer, Neues Mag. Bot.:90. 1794. $1 \rightarrow$ Arcyria denudata (L.) Wettst.

Arcyria similis Racib., in Rozpr. Mat. Przyr. Ak. Krak.:81. 1884. $1 \rightarrow$ Arcyria affinis Rostaf. emend. Nann.-Bremek. Note 2.

Badhamia hyalina (Pers.) Berk., Trans. Linn. Soc. Lond. 21:153. 1853. $1 \rightarrow *$ Badhamia capsulifera (Bull.) Berk. Trans. Linn. Soc. Lond. 21:153. 1853.

Badhamia gigantospora Ukkola \& Härk., Karstenia 36:43. 1996. 11

Badhamiopsis ainoae (Yamash.) T.E Brooks \& H.W. Keller, Mycologia 68:836. 1976. 12 (see also 10)

Calomyxa metallica (Berk.) Nieuwl., Am. Midl. Nat. 4:335. 1916. 8, 9, 12

Ceratiomyxa fruticulosa (F. Muell.) T. Macbr., N. Am. Slime-Moulds:18. 1899. 3, 4, 9, 12

Ceratiomyxa fruticulosa var. descendens Emoto, Proc. Imp. Acad. 9:416. 1933. 9

Ceratiomyxa fruticulosa var. porioides (Alb. \& Schwein.) Lister, Mycet.:26. 1894. 12

Ceratiomyxa sphaerosperma Boedijn, Misc. Zool. Sumatr. 24:1. 1927. 12

Clastoderma debaryanum A. Blytt, Bot. Zeit. 38:343. 1880. 9

Comatricha elegans (Racib.) G. Lister, Guide Brit. Mycet. ed. 3:31. 1909. 3, 4

Comatricha ellae Härk., Karstenia 17:87. 1977 (Comatricha nannengae Härk.), Karstenia 18:23. 1978 (Comatricha ellae Härk., nomen novum). 9

Comatricha laxa Rostaf., Mon.:201. 1874. 9

Comatricha longa Peck, Ann. Rep. N. Y. State Mus. 43:70. 1890. 9 (see also 10)

Comatricha nigra (Pers.) Schroet., Krypt.-Fl. Schles. 3(1):118. 1885. 1 (as C. nigra (Pers.) Preuss.), 3, 4, 12

Cornuvia circumscissa (Wallr.) Rost. var. spinosa Schroet., Krypt.-F1. Schles. 1:109. 1885. 1, 7 $\rightarrow$ Perichaena chrysosperma (Curr.) Lister ?. Note 3.

* Cornuvia serpula (Wigand) Rostaf. in Fuckel, Jahrb. Nass. Ver. Nat. 27-28:76. 1873. 1 
(*Cornuvia wrightii B. \& Br. 1. Note 4.)

Craterium aureum (Schumach.) Rostaf., Mon:124. 1874. 12

Craterium leucocephalum (Pers.) Ditmar in Sturm, Deutsch. Fl., Pilze 1:, 1:21. 1813. 3, 4, 12

Cribraria aurantiaca Schrad., Nov. Gen P1.: 5. 1797. 3, 4, 9

Cribraria cancellata (Batsch) Nann.-Bremek., Acta Bot. Neerl. 11:22. 1962. 3, 4, 9

Cribraria confusa Nann.-Bremek. \& Y. Yamam., Proc. Kon. Nederlandse Akad. Wetensch. C 86:212. 1983. 9

Cribraria microcarpa (Schrad.) Pers., Syn. Fung.:190. 1801, emend. Nann.-Bremek., Proc. Kon. Nederlandse Akad. Wetensch. C 69:340. 1966. 3, 4, 9

Cribraria minutissima Schwein., Trans. Amer. Phil. Soc. II, 4:260. 1832. 12

Cribraria violacea Rex, Proc. Acad. Nat. Sci. Philad. 43:393. 1891. 9

Diachea leucopodia (Bull.) Rostaf. Mon.:190. 1874 1, 6 (as Diachea leocopoda (Bull.) Fr.), 12. Note 5.

Dictydiaethalium plumbeum (Schumach.) Rostaf., Versuch.:5. 1873, 12

Diderma deplanatum Fr., Syst. Mycol. 3:110. 1892. 9

Diderma effusum (Schwein.) Morgan, J. Cinc. Soc. Nat. Hist. 16: 155. 1894. 3, 4, 9, 12

Diderma hemisphaericum (Bull.) Hornem., Fl. Dan. 33:13. 1829. 3, 4, 8, 9, 12

Diderma rugosum (Rex) T. Macbr., N. Am. SlimeMoulds:105. 1899. 9

Didymium anellus Morgan, J. Cinc. Soc. Nat. Hist. 16:148. 1894. 12

Didymium bahiense Gottsb., Nowa Hedwigia 15:365. 1968. 3, 4, 12 (see also 10)

Didymium clavus (Alb. \& Schwein.) Rabenh., Deuts. Krypt.-F1. 1:280. 1844. 12

Didymium difforme (Pers.) S. F. Gray, Nat. Arr. Brit. Pl. 1:571. 18212

Didymium iridis (Ditmar) Fr., Syst. Myc. 3:120; 1829. 9 (see also $\mathbf{1 0}, \mathbf{1 2}$ )

Didymium leoninum Berk. \& Broome, J. Linn. Soc. 14:83. 1873. 9

Didymium minus (Lister) Morgan, J. Cinc. Soc. Nat. Hist. 16:145. 1894. 3, 4, 12

Didymium nigripes (Link) Fr., Syst. Mycol. 3:119. 1829. 3, 4, 9, 12 (see also $\mathbf{1 0}$ )

Didymium squamulosum (Alb. \& Schwein.) Fr., Symb. Gast.: 119. 1818. 1, 3, 4, 8, 9, 12

Didymium verrucosporum A.L. Welden, Mycologia 46:98. 1954. 2

Echinostelium minutum de Bary, in Rostaf., Mon.:215. 1874. 9, 12

Enerthenema papillatum (Pers.) Rostaf., Mon. App.:28. 1876. 9

Enteridium intermedium (Nann.-Bremek.) M.L. Farr, Taxon 25:514. 1976. $9 \rightarrow$ Reticularia intermedia Nann.-Bremek., Med. Bot. Mus. Utrecht 149: 773. 1958. Note 6.

Fuligo cf. cinerea (Schwein.) Morgan, J. Cinc. Soc. Nat. Hist. 19:33. 1896. 12. Note 7.

Fuligo septica (Link) Gmel. Syst. Nat.:1466. 1791. $5 \rightarrow$ Fuligo septica (L.) F.H. Wigg.
Fuligo septica (L.) F.H. Wigg. Prim. Fl. Holsat.:112 1780. 3, 4, 9, 12

Fuligo tatrica Racib., Hedwigia 24:169. 1885. $11 \rightarrow$ Fuligo septica (L.) F.H. Wigg.

Hemiarcyria clavata (Pers.) Rost. Mon.:264. 1875. 1, 5, $6 \rightarrow$ (* Hemitrichia clavata (Pers.) Rostaf., in Fuckel, Jahrb. Nass. Ver. Nat. 27-28:75. 1873.) Note 8.

Hemitrichia calyculata (Speg.) M.L. Farr, Mycologia 66:887. 1974. 3, 4, 9, 12 (see also 10)

Hemitrichia serpula (Scop.) Rostaf., Versuch.:14. 1873. 9

Lamproderma arcyrionema Rostaf., Mon.:208 1874. 3, 4, 9, 12

Lamproderma biasperosporum Kowalski, Mycologia 60:758. 1968. 9 (see also 10)

Lamproderma scintillans (Berk. \& Broome) Morgan, J. Cinc. Soc. Nat. Hist. 16:131. 1894. 3, 4, 9, 12

Leptoderma iridescens G. Lister, J. Bot. Lond. 51:1. 1913. 12

Licea alexopouli M. Blackw., Proc. Iowa. Acad. Sci. 81:6. 1974. 2

Licea biforis Morgan, J. Cinc. Soc. Nat. Hist. 15:131. 1893. 8, 9, 12

Licea bulbosa Nann.-Bremek. \& Y. Yamam., Proc. Kon. Nederlandse Akad. Wetensch. C 0:324. 1987. 12

Licea kleistobolus G.W. Martin, Mycologia 34:702. 1942. 8, 9

Licea opercula (Wingate) G.W. Martin, Mycologia 34:702. 1942. 9

Licea parasitica (Zugal) G.W. Martin, Mycologia 34:702. 1942. 8, 12

Licea cf. pedicellata (H.C. Gilbert) H. C. Gilbert, Mycologia 34: 702. 1942. 12. Note 9.

Licea poculiformis Ukkola, Acta Bot. Fennica 160:5. 1998. 9

Licea tanzanica Ukkola, Härk. \& Gilert, in Ukkola et al., Karstenia 36:57. 1996. 9, 12

*Licea variabilis Schrad., Nov. Gen. Pl.:18. 1797. 1

Lycogala epidendrum (L.) Fr., Syst. Mycol. 3:80. 1829. 1, 3, 4, 9, 12 .

Lycogala exiguum Morgan, J. Cinc. Soc. Nat. Hist. 15:134. 1893. 3, 4, 9

Macbrideola martinii (Alexop. \& Beneke) Alexop., Mycologia 59:114. 1967. 9

Metatrichia floriformis (Schwein.) Nann.-Bremek., Proc. Kon. Nederlandse Akad. Wetensch. C 85:556. 1982. 3, 4, 9

Metatrichia horrida Ing, Trans. Brit. Mycol. Soc. 47:51. 1964. 3, 4

Metatrichia vesparium (Batsch) Nann.-Bremek., Proc. Kon. Nederlandse Akad. Wetensch. C 69:348. 1966. 9

Paradiachea cylindrica (Bilgram) R.J.G. Hertel, Dusenia 7:349. 1956. 3, 4

Paradiacheopsis longipes Hoof \& Nann.-Bremek., Proc. Kon. Nederlandse Akad. Wetensch. C 99:51. 1996. 9

Paradiacheopsis rigida (Brândză) Nann.-Bremek., Proc. Kon. Nederlandse. Akad. Wetensch. C 7:209. 1967. 9

Perichaena chrysosperma (Curr.) Lister, Mycet.:196. 1894. 2 (reference to 7), 8, 9, 12 
Perichaena corticalis (Batsch) Rostaf., Mon.:293. 1875. 8, 9, 12

Perichaena corticalis (Batsch) Rostaf. var. liceoides Lister, Mycetozoa, ed. 2:251. 1911. 12

Perichaena depressa Lib., Pl. Crypt.:378. 1837. 2, 8, 9, 12

Perichaena cf. liceoides Rost., Mon:295. 1875. $2 \rightarrow$ Perichaena corticalis Rostaf. var. liceoides Lister. Note 10.

Perichaena syncarpon T.E. Brooks, Mycologia 38:110. 1946. 2

Physarella oblonga (Berk. \& M.A. Curtis) Morgan, J. Cinc. Soc. Nat. Hist. 19:7. 1896. 3, 4

Physarum auriscalpium Cooke, Ann. Lyc. N. Y. 11:384. 1877. 12

Physarum bitectum G. Lister, Mycet. ed. 2.:78. 1911. 12

Physarum bogoriense Racib., Hedwigia 37: 52. 18 F. 1898. 3, 4, 9

Physarum citrinum Schumach., Enum. Pl. Saell. 2:201. 1803. 3, 4

Physarum compressum Alb. \& Schwein., Consp. Fung.: 97. 1805. 1 (misidentification), 3, 4, 9, 12. Note 11.

Physarum crateriforme Petch, Ann. R. Bot. Gard. Peradeniya 4:304. 1909. 8, 12

Physarum diderma Rostaf., Mon.:110. 1874. 8, 12

Physarum echinosporum Lister, J. Bot. Lond. 37:147. 1899. 8

Physarum fulgens Pat., Bull. Soc. Myc. France 8:12. 1892. 9

Physarum globuliferum (Bull.) Pers., Syn. Fung.:175. 1801. 9

Physarum javanicum Racib., Hedwigia 37:53. 1898. $3,4,12$

Physarum lakhanpalii Nann.-Bremek. \& Y. Yamam., Proc. Kon. Nederlandse Akad. Wetensch. C 90:335. 1987. 9

Physarum leucophaeum Fr., Symb. Gast.:24. 1818. 1 (with a question mark), 8,9

Physarum melleum (Berk. \& Broome) Massee, Mon.:278. 1892. 3, 4, 9, 12

Physarum mutabile (Rostaf.) G. Lister, in Lister, Mycet. ed. 2:53. 1911. 3, 4, 9, 12

Physarum notabile T. Macbr., N. Am. Slime-Moulds ed. 2:80. 1922. 3, 4, 9

Physarum nucleatum Rex, Proc. Acad. Phila. 43:389. 1891. 3, 4, 9, 12

Physarum nutans Pers., Ann. Bot. Usteri 15:6. 1795. $3,4,9$

Physarum oblatum T. Macbr., Bull. Nat. Hist. Univ. Iowa 2:384. 1893. 8, 9

Physarum ovisporum G. Lister, J. Bot. Lond. 59:90. 1921. 12 (see also 10)

Physarum perfectum M.E. Peck, in Peck \& Gilbert, Am. Jour. Bot. 19:134. 1932. 12

Physarum pezizoideum (Jungh.) Pavill. \& Lagarde, Bull. Soc. Myc. Fr. 19:87. 1903. 9, 11

Physarum pezizoideum var. pezizoideum $3,4,11 \rightarrow$ Badhamia gigantospora Ukkola \& Härk.

Physarum pezizoideum var. microsporum M.L. Farr, Brittonia 16:340. 1964. 3, 4, $11 \rightarrow$ Physarum pezizoideum (Jungh.) Pavill. \& Lagarde. Note 12.
Physarum psittacinum Ditmar, in Sturm, Deuts. F1. Pilze 1:125. 1817. 3, 4

Physarum pusillum (Berk. \& M.A. Curtis) G. Lister, Mycet. ed. 2:64. 1911. 3, 4, 8, 9, 12

Physarum spumarioides T.N. Lakh. \& Mukerji var. degawae Nann.-Bremek. \& Y. Yamam., Proc. Kon. Nederlandse Akad. Wetensch. C 93:275. 1990. 8

Physarum stellatum (Massee) G.W. Martin, Mycologia 39:461. 1947. 12

Physarum superbum Hagelst., Mycologia 32:385. 1944. 3, 4

Physarum vernum Sommerf., in Fries, Syst. Mycol. 3:146. 1829. 9, 12 (see also 10)

Physarum cf. virescens Ditmar, in Sturm, Deuts. Fl. Pilze 1:123. 1817. 12. Note 13.

Physarum viride (Bull.) Pers., Ann. Bot. Usteri 15:6. 1795. 3, 4, 9

Stemonitis axifera (Bull.) T. Macbr., N. Am. SlimeMoulds:120.1889. 3, 4, 9, 12

Stemonitis fusca Roth, Mag. Bot. Römer \& Usteri 1:26. 1787. 2 (reference to 7 ), $\mathbf{3}, \mathbf{4}, \mathbf{5}, \mathbf{7}, \mathbf{9}, \mathbf{1 2}$

Stemonitis pallida Wingate, in T. Macbr. N. Am. Slime-Moulds:123. 1899. 3, 4, 9

Stemonitis smithii T. Macbr., Bull. Nat. Hist. Univ. Iowa 2:381. 1893. 3, 4

Stemonitis splendens Rostaf., Mon.:195. 1874. 3, 4, 9, 12

Stemonitopsis gracilis (G. Lister) Nann.-Bremek., Proc. Kon. Nederlandse Akad. Wetensch. C. 76: 486. 1973. 9

Stemonitopsis microspora (Lister) Nann.-Bremek., Guide Temp. Myxom.:339. 1991. 3, 4

Stemonitopsis typhina (F.H. Wigg.) Nann.-Bremek., Guide Temp. Myxom.:341. 1991. 3, 4, 9

Tilmadoche nutans (Pers.) Rost. Mon.:127. 1874. $6 \rightarrow$ Physarum nutans Pers.

Tilmadoche viridis (Gmel.) Sacc., Michelia 2:263. 1881. $1 \rightarrow$ Physarum viride (Bull.) Pers.

Trichia botrytis (J.F. Gmel.) Pers., Neues Mag. Bot. 1:89. 1794. 12

Trichia decipiens T. Macbr., N. Am. Slime-Moulds: 218. 1899.9

Trichia favoginea (Batsch) Pers., Neues Mag. Bot. 1:90. 1794. 3, 4

Trichia stuhlmanni Eichelb., Verh. Nat. Ver. Hamburg 3:32. 1906. $1 \rightarrow$ Trichia decipiens T. Macbr. Note 14.

Tubifera bombarda (Berk. \& Broome) G.W. Martin, Brittonia 13:110. 1961. 3, 4, 9

Tubifera microsperma (Berk. \& M.A. Curtis) G.W. Martin, Mycologia 39:461. 1947. 3, 4

\section{Notes}

Note 1. This taxon has not been listed by Eichelbaum (1906), but he has collected it from Bomole, near Ama$\mathrm{ni}$. The specimen is one of his five myxomycete collections from Tanzania still left in HBG (Hamburg, Germany), and has been seen by the author. Eichelbaum's collection has been reported by Hennings (1907).

Note 2. Lister (1925) considers this taxon to be a synonym of Arcyria incarnata Pers. var. fulgens G. Lister. 
According to Nannenga-Bremekamp (1991), Arcyria affinis is a very variable species, and she considers $A$. similis Rostaf. to be a form of $A$. affinis (with very thick capillitial tubes ornamented with an intricate pattern of thickenings). Since the specimen reported by Eichelbaum (1906) is not available, it is not possible to confirm the identification, but in this check-list the circumscription of $A$. affinis follows Nannenga-Bremekamp (1991).

Note 3. The identity of this taxon is slightly doubtful, but according to Martin and Alexopoulos (1969) it probably is Perichaena chrysosperma, since the description of the variety spinosa is based on capillitial characters. Cornuvia circumscissa (Wallr.) Rostaf. is a possible synonym of Perichaena depressa (Martin \& Alexopoulos 1969).

Note 4. I have not found the publication where Cornuvia wrightii B. \& Br. was published. The authors mentioned by Eichelbaum probably mean Berkeley and Broome. Ophiotheca wrightii was described by Berkeley and M.A. Curtis in 1869, and later by Rostafiński removed to the genus Cornuvia (Rostafiński 1876, Lister 1925, Martin \& Alexopuolos 1969). According to Lister (1925) and Martin and Alexopoulos (1969), Cornuvia wrightii (Berk. \& M.A. Curtis) Rostaf. is a synonym of Perichaena chrysosperma (Curr.) Lister. The identity of the taxon reported by Eichelbaum (1906) is doubtful, since he also mentions Cornuvia circumscissa (Wallr.) Rost. var. spinosa Schroet. from Tanzania (see previous note).

Note 5. The specific epithet was spelled leucopodia by Bulliard, but Fries (1829) cited it as leucopoda, which was followed e.g. by Rostafiński (1874) and Lister (1925). According to Martin and Alexopoulos (1969) there is no orthographical reason for the change of Bulliard's spelling.

Note 6. Farr (1976) considered Reticularia Bull. as a later homonym of Reticularia Baumg. (lichens), and proposed its replacement with Enteridium Ehrenb., the next available generic name. Lado et al. (1998) have further clarified the taxonomical status of Reticularia (Bull.), and according to them it was validly published by 1788 and has priority over Reticularia Baumg. published in 1790. Lado and Pando (1998) have proposed the conservation of the name Reticularia Bull. with a conserved type.

Note 7. The crowded, heaped fructifications growing on dead banana leaves resemble Fuligo cinerea without a cortex. In some respect they also resemble Physarum didermoides (Pers.) Rostaf., and Badhamia cinerascens G.W. Martin. The former species, however, has a double peridium and often angular or irregularly shaped spores, the latter has spherical or nearly spherical spores (Martin \& Alexopoulos 1969). The Tanzanian specimen looks similar to that illustrated in the figure-table (no. 75) of F. cinerea in Lister's monograph (1925).

Note 8. No herbarium specimens of these collections from Tanzania are available. Hemitrichia clavata is considered to be a temperate species and according to Mar- tin and Alexopoulos (1969) all tropical collections referred to $H$. clavata which they have seen, have proved to be Hemitrichia calyculata (Speg.) M. L. Farr. Hemitrichia clavata, however, has been reported by Almeida (1974) from Mocambique, and by Rammoloo and Mitchell (1994) from Malawi. Hemitrichia calyculata is by some authors considered only to be a variety of H. clavata (see e.g. Lister 1925, Hagelstein 1944, Yamamoto et al. 1993).

Note 9. The scanty specimen developed in a moist chamber culture on the bark of Cupressus lusitanica approaches $L$. pedicellata, but compared to the type specimen (H.C. Gilbert 2117, Milford, lowa, 16.VII.1932) the Tanzanian specimen has smaller and totally smooth spores.

Note 10. Lister (1925) and Hagelstein (1944) considered Rostafiński's Perichaena liceoides to be a variety of Perichaena corticalis. Gilert (1990) studied the type collection and agreed. Eliasson and Lundqvist (1979) cultivated many dung samples originating from different parts of the world, one that produced this taxon was collected in Tanzania (cow dung). The fructifications developed in moist chamber cultures showed a great variation from aggregated yellowish brown to scattered dark brown sporangia; there were, however, specimens which represented transitional forms between these extremes (Eliasson \& Lundvqist 1979).

Note 11. Eichelbaum (1906) reported two collections, which are still deposited in HBG, from Amani. The collections were found in the autumn of 1997, and sent to the author. Both proved to be Badhamia gigantospora Ukkola \& Härk. (see Ukkola \& Härkönen 1996). Eichelbaum had identified the specimens with a question mark, which, however, were not included in his publication.

Note 12. The taxonomic status of Physarum pezizoideum var. pezizoideum and var. microsporum was reexamined by Ukkola and Härkönen (1996). Checking the type material of Trichamphora pezizoidea Jungh. showed that var. microsporum is a synonym of var. pezizoideum, while var. pezizoideum sensu Farr belongs to a distinct species, described as Badhamia gigantospora (type from Tanzania).

Note 13. The Tanzanian specimen macroscopically resembles Physarum virescens, but the spores are smaller and paler. Physarum obscurum (Lister) Ing has spores of similar size as the Tanzanian specimen, but differs in having scattered rather than heaped sporangia, the colour is olivaceous rather than greenish-yellow, and the capillitium contains limeless theads, producing a more rigid network (Ing 1982).

Note 14. Eichelbaum (1906) described T. stuhlmanni as a new species from Tanzania. Lister (1925) and Martin and Alexopoulos (1969) regard it as a synonym of Trichia decipiens T. Macbr. The description given by Eichelbaum (1906) is not very detailed. All characteristics mentioned, except the colour of the sporangia (dark red-brown), fit the Tanzanian specimens of $T$. decipiens studied by the author (see Ukkola 1998b). 
Acknowledgements. I wish to thank Prof. Uno Eliasson (University of Göteborg, Sweden) for reviewing the manuscript, and Donald Smart for checking the English.

\section{References}

Almeida, M.G. 1974: Contribuição para o conhecimento dos Myxomycetes de Moçambique. - Bol. Soc. Brotheriana 48: 205-210.

1 Eichelbaum, F. 1906: Beiträge zur Kenntnis der Pilzflora des Ostusambaragebirges. - Verh. Naturwiss. Ver. Hamburg 3: 1-92.

2 Eliasson, U. \& Lundqvist, N. 1979: Fimicolous myxomycetes. - Bot. Notiser 132: 551-568.

Farr, M.L. 1976: Reticularia Baumgarten (lichens) versus Reticularia Bulliard (Myxomycetes). - Taxon 25: 514.

Fries, E. 1829: Systema Mycologicum, sistens fungorum ordines, genera et species. 3. - Greifswald. 524 pp.

Gilert, E. 1990: On the identity of Perichaena liceoides (Myxomycetes). - Mycol. Res. 94: 698-704.

Hagelstein, R. 1944: The Mycetozoa of North America. - Lancaster Press, Lancaster. 306 pp.

3 Härkönen, M. \& Saarimäki, T. 1991: Tanzanian Myxomycetes: first survey. - Karstenia 31: 41-54.

Härkönen, M. \& Saarimäki, T. 1992: Tanzanian Myxomycetes: first survey (addition). - Karstenia 32: 6.

4 Härkönen, M. \& Saarimäki, T. 1994: Myxomycetes, an unexplored class of fungi in Tanzania. - In: Seyani, J.H. \& Chikuni, A.C. (eds.), Proc. XIIIth Plenary Meeting AETFAT 1: 735-748. Zomba, Malawi. 793 pp.

5 Hennings, P. 1905: Fungi Africae orientalis. III. Englers bot. Jahrb. 34: 39-57.

6 Hennings, P. 1907: Fungi Africae orientalis. IV. Englers bot. Jahrb. 38: 102-118.

Ing, B. 1982: Notes on Myxomycetes 3. - Trans. British Mycol. Soc. 78: 439-446.

Ing, B. 1999: The Myxomycetes of Britain and Ireland. An Identification Handbook. - The Richmond Publishing Co. Ltd. 374 pp.

Iversen, S.T. 1991: The Usambara Mountains, NE Tanzania: history, vegetation and conservation. - Uppsala Universitetet, Uppsala. 143 pp.

Kirk, P.M. \& Ansel, A.E. 1992: Authors of Fungal Names. Index of fungi supplement. - International Mycological Institute C.A.B. International, Wallingford. $95 \mathrm{pp}$.

Lado, C. \& Pando, F. 1998: (1340) Proposal to conserve the name Reticularia (myxomycetes) with a conserved type. - Taxon 47: 453-454.

Lado, C., Pando, F. \& Rico, V. 1998: The nomenclatural status of Reticularia (myxomycetes). - Taxon 47: 109-111.

Lister, A. 1911: A monograph of the Mycetozoa. $2^{\text {nd }}$ ed., revised by G. Lister. - British Museum, London. 302 pp., $200 \mathrm{pl}$.
Lister, A. 1925: A monograph of the Mycetozoa. $3^{\text {rd }}$ ed., revised by G. Lister. - British Museum, London. $296 \mathrm{pp}$.

Martin, G.W. \& Alexopoulos, C.J. 1969: The Myxomycetes. - Univ. Iowa Press, Iowa City. 560 pp.

Martin, G.W., Alexopoulos, C.J. \& Farr, M.L. 1983: The genera of Myxomycetes. - Univ. Iowa Press, Iowa City. 102 pp., 41 pls.

Nannenga-Bremekamp, N.E. 1967: Notes on Myxomycetes XII. A revision of the Stemonitales. - Proc. Kon. Nederlandse Akad. Wetensch. C 7: 201-216.

Nannenga-Bremekamp, N.E. 1991: A guide to temperate Myxomycetes. - Biopress, Bristol. 409 pp. (Engl. transl. by A. Feest and Y. Burgraaf of De Nederlandse Myxomyceten. 1974, 409 pp., Kon. Nederlandse Nat. Ver., Zutphen.)

Neubert, H., Nowotny, W. \& Baumann, K. 1993: Die Myxomyceten Deutschlands und des angrenzenden Alpenraumes unter besonderer Berücksichtigung Österreichs 1. Ceratiomyxales, Echinosteliales, Liceales, Trichiales. - Karlheinz Baumann Verlag, Gomaringen. 343 pp.

Neubert, H., Nowotny, W., Baumann, K. \& Marx, H. 1995: Die Myxomyceten Deutschlands und des angrenzenden Alpenraumes unter besonderer Berücksichtigung Österreichs 2. Physarales. - Karlheinz Baumann Verlag, Gomaringen. 368 pp.

Rammeloo, J. \& Mitchell, D.W. 1994: Contribution towards the knowledge of the Myxomycetes of Malawi and Zambia. - In: Seyani, J.H. \& Chikuni, A.C. (eds.), Proc. XIIIth Plenary Meeting AETFAT 1: 785-793. Zomba, Malawi. 793 pp.

Rostafiński, J. 1874-1876: Śluzowce (Mycetozoa) monografia. - Pamiet. Towarz. Nauk. Sci. Pary 5(4): 1-215 (1874); 6(1): 216-432 (1875); Dodatek (Supplement) 8(4): 1-42 (1876).

7 Schmidt, A. 1913: Beitrag zur Kenntnis der deutschostafrikanischen Mistpilze. - Jahresber. Schles. Ges. Vaterl. Cultur, I 2 Abt. b. Zool.-Bot. Sekt. pp. 17-25.

8 Ukkola, T. 1998a: Some Myxomycetes from Dar es Salaam (Tanzania) developed in moist chamber cultures. - Karstenia 38: 27-36.

9 Ukkola, T. 1998b: Myxomycetes of the Usambara Mountains, NE Tanzania. - Acta Bot. Fennica 160: $1-37$.

10 Ukkola, T. 1998c: Tanzanian Myxomycetes to the end of 1995. - Publ. Bot. Univ. Helsinki 27: 1-45.

11 Ukkola, T. \& Härkönen, M. 1996: Revision of Physarum pezizoideum var. pezizoideum and var. microsporum (Myxomycetes). - Karstenia 36: 41-46.

12 Ukkola, T., Härkönen, M. \& Saarimäki, T. 1996: Tanzanian Myxomycetes: second survey. - Karstenia 36: $51-77$.

Yamamoto, Y., Hagiwara, H. \& Sultana, K. 1993: Myxomycetes from Northern Pakistan 2. - Cryptog. Fl. Pakistan 2: $25-41$. 